\title{
Clinical importance of neuroimaging in epilepsy
}

Epilepsy is a chronic condition throughout the world, characterized by recurrent seizures with no acute warning. Epilepsy is one of the most frequently seen neurological diseases, with an incidence of between 5 and 8 per 1000 in the general population. In children below the age of 16 years, epilepsy is seen at a rate of $0.5-1 \%$, with the highest incidence rate in children below the age of 1 year. ${ }^{[1,2]}$

For a patient who presents with a history of seizures, after physical and neurological evaluations, electroencephalography, biochemical and metabolic evaluations, computerized brain tomography (CT) and magnetic resonance imaging (MRI) should be planned according to the clinical priority of the patient.

The basic role of radiological imaging in epilepsy is to define any structural anomalies or pathologies and to aid in the patient treatment protocol. In the majority of studies in industrialized countries, a specific etiology has been defined in $60-70 \%$ of cases. However, despite there being greater risk factors in developed countries, rates of specific etiology have been reported of $40 \%$ and lower. The etiological differentiation in these studies has generally been dependent on the clinical data, but if techniques are used, which are provided by advanced technology, an increase in the rates of specific etiology can be expected. ${ }^{[3,4]}$

Depending on the level of development of the country, etiological differences, central nervous system infections and cerebrovascular events are seen. ${ }^{[1,5]}$ The most common etiologies in those aged under 15 years have been found to be congenital structural defects, trauma, and hypoxic ischemic encephalopathy. ${ }^{[2]}$

Within the last 10-15 years, significant advances have been made in imaging techniques. Intracranial

\begin{tabular}{|l|l|}
\hline \multicolumn{2}{|c|}{ Access this article online } \\
\hline Quick Response Code: & Website: \\
\hline & www.ruralneuropractice.com \\
\cline { 2 - 2 } & \\
\hline
\end{tabular}

anatomical structures clearly began to image. Some functional areas in the brain can be detected via imaging. The basic radiological modalities in cases with epilepsy are CT and MRI methods; partially ultrasonography (US) and digital subtraction angiography (DSA). In the preoperative evaluation of patients with resistant epilepsy who are candidates for surgery, single photon emission computerized tomography (SPECT), positron emission tomography (PET), magnetic resonance spectroscopy (MRS), functional magnetic resonance (fMR), and magneto-encephalography (MEG) are other imaging methods which are used.

US is widely used as it is easily applied to newborns, is repeatable, and does not contain ionizing radiation. In premature babies, it is useful in the determination of pathologies such as intracranial bleeding, hydrocephaly, and congenital anomalies. ${ }^{[6,7]}$

The advantages of CT are that it is cheap, easily accessible, easy to apply, and the duration of taking the images is short. However, with the advent of MRI, the importance of CT has reduced, although it is still an imaging method which can be used in the first evaluation of seizures related to trauma, emergency situations, fever, or focal neurological changes. CT may be useful in pathologies including hemorrhage, infarct, Galen vein malformation, ventricular dilation, and calcification. It should be selected for hemorrhage, hydrocephaly, and major structural pathologies in both children and adults. CT can show approximately 50\% of lesions such as vascular malformation and tumors causing epilepsy. In cases with characteristic CT findings such as tuberosclerosis and Sturge-Weber syndrome, noncalcified lesions (e.g., tubers) can be determined by MRI. The presence of bone artifacts in the temporal fossa, particularly when combined with the low-tissue contrast property of $\mathrm{CT}$, makes it impossible to determine mesial temporal sclerosis (MTS). In conclusion, it can be said that CT should be preferred in conditions which cannot be performed or found by MRI. In an epileptic case which is normal on CT, it is necessary to perform MRI. With new developments in MRI technology, some previously undefined structural anomalies can now be easily determined. When MRI is compared with CT, high sensitivity, excellent soft-tissue contrast, the possibility of 
multiplanar imaging and the fact that it does not contain ionizing radiation have made MRI the leading method to be used in the evaluation of epileptic patients. ${ }^{[8,9]}$

MRI plays an important role in epilepsy surgery in the definition of structural anomalies and pathologies and at the same time in the evaluation of the relationships between lesions and functional areas such as motor, speech, and memory areas. As the pathology defined by MRI conforms to clinical and electrophysiological data, it is important in terms of preventing false-positive results. The conformity of MRI findings with noninvasive electrophysiological data removes the necessity to apply invasive electroencephalographic imaging. ${ }^{[10]}$

Postoperative follow-up MRIs can define residue or recurrent lesions and evaluate the adequacy of the surgical treatment. Being able to make an accurate postoperative evaluation of epileptogenic foci facilitates the follow-up of seizure prognosis. ${ }^{[11]}$

In conclusion, the development of new imaging techniques every year gives us the opportunity to obtain additional radio-anatomic or physiopathological information. Therefore, it is thought that some of the above-written information of epilepsy patients will be revised in the near future.

\author{
Mustafa Calik, Ekrem Karakas ${ }^{1}$, Nesat Cullu ${ }^{1}$ \\ Department of Pediatric Neurology, Harran University School \\ of Medicine, Sanliurfa, ${ }^{1}$ Radiology, Harran University School of \\ Medicine, Sanliurfa, Turkey
}

Address for correspondence:

Dr. Mustafa Calik,

Department of Pediatric Neurology,

Harran University School of Medicine, Sanliurfa, Turkey.

E-mail:m.calik80@hotmail.com

\section{References}

1. Hauser WA, Annegers JF, Kurland LT. Prevalence of epilepsy in Rochester, Minnesota, 1940-1980. Epilepsia 1991;32:429-45.

2. Arzimanogolou A, Guerrini R, Aicardi J. Epilepsy in children. $3^{\text {rd }}$ ed. Philadelphia: Lippincott Williams and Wilkins; 2004.

3. Senanayake N, Roman GC. Epidemiology of epilepsy in developing countries. Bull World Health Organ 1993;71:247-58.

4. Bakhsh A. Value of neuroimaging in epilepsy-an experience from Pakistan. J Neurosc Rural Pract 2013;4(Suppl 1):s35-9.

5. Rwiza HT, Kilonzo GP, Haule J, Matuja WB, Mteza I, Mbena P, et al. Prevalence and incidence of epilepsy in Ulanga, a rural Tanzanian district: A community-based study. Epilepsia 1992;33:1051-6.

6. Fisher RS, Stein A, Karis J. Epilepsy fort the neuroradiologist. Am J Neuroradiol 1997;18:851-3.

7. Franz DN, Ball WS Jr. A Clinical approach to imaging in childhood epilepsia. In: Ball WS Jr editor. Pediatric Neuroradiology. Philadelphia: Lippincott-Raven; 1997. p. 489-504.

8. Kuzniecky RI, Knowlton RC. Neuroimaging of epilepsy. Semin Neurol 2002;22:279-88.

9. Velez-Ruiz NJ, Klein JP. Neuroimaging in the evaluation of epilepsy. Semin Neurol 2012;32:361-73.

10. Miserocchi A, Cascardo B, Piroddi C, Fuschillo D, Cardinale F, Nobili L, et al. Surgery for temporal lobe epilepsy in children: Relevance of presurgical evaluation and analysis of outcome. J Neurosurg Pediatr 2013;11:256-67.

11. Bronen RA, Fulbright RK, King D, Kim JH, Spencer SS, Spencer DD, et al. Qualitative MR imaging of refractory temporal lobe epilepsy requiring surgery: Correlation with pathology and seizure outcome after surgery. Am J Roentgenol 1997;169:875-82.

How to cite this article: Calik M, Karakas E, Cullu N. Clinical importance of neuroimaging in epilepsy. J Neurosci Rural Pract 2013, 4(Suppl 1):s11-2.

Source of Support: Nil. Conflict of Interest: None declared. 